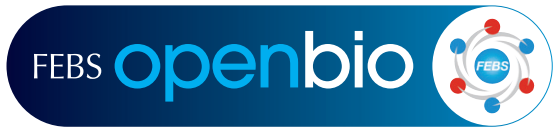

\title{
Crystal structure of human nicotinic acid phosphoribosyltransferase
}

\author{
Ada Serena Marletta ${ }^{a}$, Alberto Massarotti ${ }^{a}$, Giuseppe Orsomando ${ }^{b}$, Giulio Magni ${ }^{\text {b }}$, Menico Rizzi ${ }^{\text {a,* }}$, \\ Silvia Garavaglia ${ }^{\mathrm{a}, *}$ \\ ${ }^{a}$ Department of Pharmaceutical Sciences, University of Piemonte Orientale, Largo Donegani 2, 28100 Novara, Italy \\ ${ }^{\mathrm{b}}$ Department of Clinical Sciences, Section of Biochemistry, Polytechnic University of Marche, Via Ranieri 67, 60131 Ancona, Italy
}

\section{A R T I C L E I N F O}

\section{Article history:}

Received 15 April 2015

Revised 30 April 2015

Accepted 1 May 2015

\section{Keywords:}

Nicotinic Acid

Preiss-Handler pathway

NAD biosynthesis

Phosphoribosyltransferase

FK866

Recycling NAD pathway

\begin{abstract}
A B S T R A C T
Nicotinic acid phosphoribosyltransferase (EC 2.4.2.11) (NaPRTase) is the rate-limiting enzyme in the three-step Preiss-Handler pathway for the biosynthesis of NAD. The enzyme catalyzes the conversion of nicotinic acid (Na) and 5-phosphoribosyl-1-pyrophosphate (PRPP) to nicotinic acid mononucleotide ( $\mathrm{NaMN}$ ) and pyrophosphate (PPi). Several studies have underlined the importance of NaPRTase for NAD homeostasis in mammals, but no crystallographic data are available for this enzyme from higher eukaryotes. Here, we report the crystal structure of human NaPRTase that was solved by molecular replacement at a resolution of $2.9 \AA$ in its ligand-free form. Our structural data allow the assignment of human NaPRTase to the type II phosphoribosyltransferase subfamily and reveal that the enzyme consists of two domains and functions as a dimer with the active site located at the interface of the monomers. The substrate-binding mode was analyzed by molecular docking simulation and provides hints into the catalytic mechanism. Moreover, structural comparison of human NaPRTase with the other two human type II phosphoribosyltransferases involved in NAD biosynthesis, quinolinate phosphoribosyltransferase and nicotinamide phosphoribosyltransferase, reveals that while the three enzymes share a conserved overall structure, a few distinctive structural traits can be identified. In particular, we show that NaPRTase lacks a tunnel that, in nicotinamide phosphoribosiltransferase, represents the binding site of its potent and selective inhibitor FK866, currently used in clinical trials as an antitumoral agent.

(c) 2015 The Authors. Published by Elsevier B.V. on behalf of the Federation of European Biochemical Societies. This
\end{abstract} is an open access article under the CC BY license (http://creativecommons.org/licenses/by/4.0/).

\section{Introduction}

Nicotinamide adenine dinucleotide (NAD) and its phosphorylated form (NADP) are essential ubiquitous coenzymes playing fundamental roles in living cells. Beyond renown redox roles in energy metabolism, $N A D(P)$ is also intimately involved in signaling pathways through a number of consuming reactions that underscore a wealth of physiopathological conditions [1-4]. Indeed, the $\mathrm{NAD}(\mathrm{P})$ derivatives nicotinic acid adenine dinucleotide phosphate

Abbreviations: Na, nicotinic acid; NaAD, nicotinic acid dinucleotide; NAD, nicotinamide adenine dinucleotide; NaMN, nicotinic acid mononucleotide; NamR, nicotinamide riboside; NaPRTase, nicotinic acid phosphoribosyltransferase; NMN, nicotinamide mononucleotide; NMNAT, nicotinamide mononucleotide adenylyltransferase; PRPP, 5-phosphoribosyl-1-pyrophosphate; QA, quinolinic acid

* Corresponding authors at: Department of Pharmaceutical Sciences, University of Piemonte Orientale, Via Bovio 6, 28100 Novara, Italy. Tel.: +39 0321 375712; fax: +390321 375821 (M. Rizzi). Tel.: +390321 375714; fax: +39 0321375821 (S. Garavaglia).

E-mail addresses: menico.rizzi@uniupo.it (M. Rizzi), silvia.garavaglia@uniupo.it (S. Garavaglia).
(NaADP) and cyclic ADP-ribose (cADPR) are among the most potent intracellular calcium-mobilizing agents [4,5]. Moreover, NAD is the substrate for poly(ADP-ribosyl)ation reactions that in higher eukaryotes regulate chromatin function and gene expression, as well as for mono(ADP-ribosyl)ation modifications of target proteins in both mammalian and prokaryotic cells [4]. In all organisms, NAD can also be consumed by important regulatory enzymes, named sirtuins, that catalyze NAD-dependent deacetylation reactions of target proteins [6]. Clearly, physiological NAD depletion caused by overall NAD-consuming reactions necessitates permanent regeneration of this cofactor. Therefore NAD biosynthesis appears of therapeutically value for the treatment of pathological conditions arising from severe altering of $\mathrm{NAD}(\mathrm{P})$ homeostasis like in the case of neurological, neoplastic, and infectious disorders, as well as in the process of ageing [1,3,7-10]. Based on current knowledge, four different substrates can be used as a source of the pyridine ring in the NAD biosynthesis: quinolinic acid (QA) in the de novo pathway; nicotinic acid $(\mathrm{Na})$, nicotinamide (Nam) and nicotinamide riboside (NamR) in the salvage pathways. While this latter compound is phosphorylated by action of an 
ATP-dependent kinase activity [11], the three other precursors, QA, $\mathrm{Na}$ and Nam, can be individually transferred to a phosphoribosyl pyrophosphate moiety (PRPP) by respective phosphoribosyltransferase activities [12]. The resulting mononucleotide products, nicotinic acid mononucleotide (NaMN) and nicotinamide mononucleotide (NMN), are then converted to dinucleotide forms, i.e. nicotinic acid dinucleotide (NaAD) and NAD, by action of a single enzymatic activity represented by nicotinamide mononucleotide adenylyltransferase (NMNAT) [13,14]. NaAD is finally amidated to NAD by a NAD synthetase activity (Fig. 1) [7,12,15].

Although the Nam salvaging biosynthetic route appears physiologically the main contributor to keep adequate NAD homeostasis [16-18], the supplementation of Nam does not seem so effective in elevating cellular NAD beyond the basal concentration. Indeed, some mammalian tissues like heart, kidney [19], and red blood cells [20] use preferentially Na for NAD synthesis even in the presence of higher Nam levels physiologically available [21]. Thus, both precursors appear relevant for NAD biosynthesis but with distinct and possibly complementary roles in different tissues [22]. This view is also supported by the observation that human embryonic kidney (HEK293) cell line, when supplemented with Na, but not with Nam, markedly elevated intracellular NAD levels, and showed beneficial effects versus the cytotoxic stress symptoms caused by $\mathrm{H}_{2} \mathrm{O}_{2}$ [22]. This effect of exogenously administered Na likely accounts for at least some effects of this vitamin precursor and suggests novel applications for the treatment of conditions associated with cellular NAD depletion, such as in photodamaged skin [23]. Of note in this context is also the finding that $\mathrm{Na}$ is one of the oldest drugs known for its unique anti-lipolytic effects [24], that has been attributed in more recent years to its specific binding to selected receptors present on the plasma membrane of adipocytes $[25,26]$. In mammals, Na can derive either directly from the diet, or indirectly from the enzymatic deamidation of dietary Nam operated by the gut flora. As shown in Fig. 1, Nicotinate Phosphoribosyltransferase (EC 2.4.2.11) is the first enzyme that catalyzes the synthesis of NaMN and PPi from Na and PRPP. The enzyme, originally named NaMN pyrophosphorylase, was first described by Handler in human erythrocytes, where it plays a key role in elevating NAD levels [20]. In both mice and humans, NaPRTase appears differentially expressed in tissues, being more abundant where $\mathrm{Na}$ is the preferential source for NAD biosynthesis [22,27]. Like the bacterial counterpart enzymes, human NaPRTase is strictly specific for $\mathrm{Na}$ as a substrate and ADP, NaAD and NAD do not affect its activity $[27,28]$. The central compounds of cellular metabolism, ATP and Pi, were found to affect human NaPRTase (hNaPRTase) activity. In particular ATP utilization seems to promote allosteric interactions between subunits [27]. Such ATP-driven conformational change can also be inferred by the observation that, in microbial NaPRTs, ATP binding protects from proteolysis [29] and heat inactivation [30].

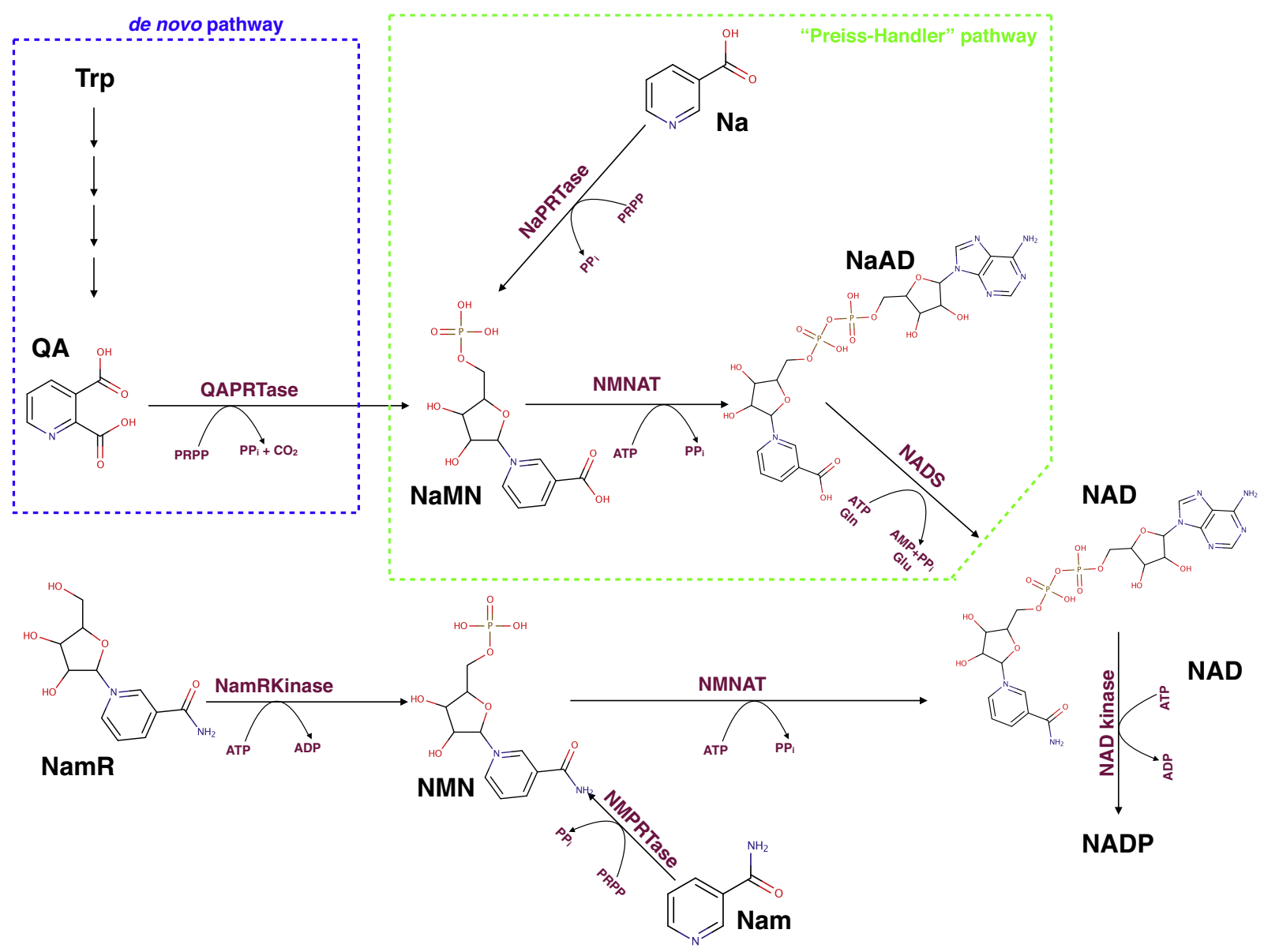

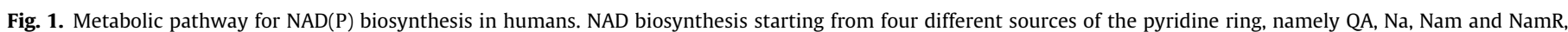

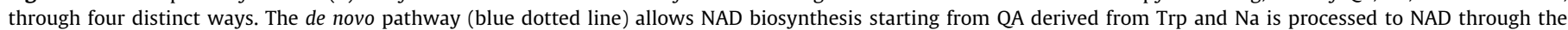

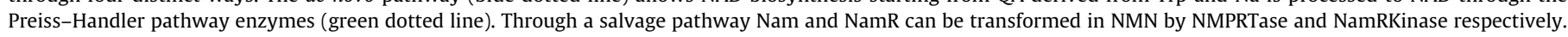

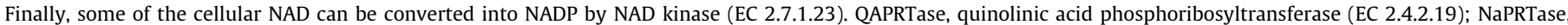

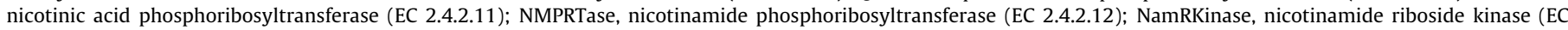
2.7.1.22); NMNAT nicotinamide mononucleotide adenylyltransferase (EC 2.7.7.1); NADS, NAD synthetase (EC 6.3.5.1). 
Although NaPRTase is not a rate-limiting enzyme for NAD synthesis in mammalian cells, several evidences indicate its complementary role in boosting and regulating NAD biosynthesis under certain conditions and in specific local districts [17]. This role appears equally relevant as that proposed for other enzymes of the same pathway, particularly in the context of possible treatments of NAD-related diseases.

Here we report on the crystal structure of the human enzyme in its free form that has been determined at a $2.9 \AA$ resolution. Such structural analysis was instrumental to (i) establish the protein folding conservation among enzymes catalyzing similar phosphoribosyltransferase reactions but using different pyridine substrates, and thus complete the structural information on the NAD salvage pathway enzymes in mammals, (ii) shed further light on the catalytic mechanism of the hNaPRTase and (iii) provide a structural basis for the lack of inhibition on NaPRTase by antitumor drugs targeting NAD biosynthesis metabolism [31]. Moreover, being the first structural report of this enzyme from a mammalian source, it may also assist future structure-based design of effectors of potential medical interest.

\section{Experimental procedures}

\subsection{Enzyme expression and purification}

The procedure adopted for the human gene cloning and bacterial overexpression of recombinant human NaPRTase has been previously reported [28] and here adjusted as follows. Induced cells collected by centrifugation were resuspended in 1/50 original vol-

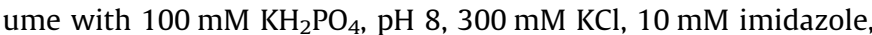
$1 \mathrm{mM} \beta$-mercaptoethanol and 250 units of benzonase nuclease. Bacterial cells membranes were fragmented by applying a high pressure through the use of the French Press twice at $1.5 \mathrm{~K}$ Bar. Protease inhibitor cocktail was added $(100 \mu \mathrm{L}$ per $40 \mathrm{~mL}$ extraction Buffer) to the crude extract that was clarified by $1 \mathrm{~h}$ centrifugation at $18,000 \mathrm{rpm}$. The supernatant was purified by a His-tag affinity chromatography and a subsequent size-exclusion chromatography. In particular, in the first purification step, the solution containing the soluble fraction of human enzyme was batch-mixed for 30 min with a Qiagen Ni-NTA resin ( $0.1 \mathrm{~mL}$ per $\mathrm{mL}$ extract), previously equilibrated with the buffer, and then packed into a chromatographic column. The flow-through and the $30 \mathrm{mM}$ imidazole buffer wash were discarded. The recombinant protein was eluted by two subsequent steps at 250 and $500 \mathrm{mM}$ imidazole. Protein concentration was determined by the Bradford protein assay. Active, SDS-PAGE homogeneous fractions, were pooled, centrifuged and loaded on a Sephacryl S200 16/60 column in a FPLC system. Elution Buffer contained $50 \mathrm{mM}$ Hepes/KOH buffer, $\mathrm{pH}$ 7.5 , and $300 \mathrm{mM} \mathrm{KCl}$ and the flow rate was $0.2 \mathrm{~mL} / \mathrm{min}$. Eluted fractions were analyzed by SDS-PAGE. All steps were performed at $4{ }^{\circ} \mathrm{C}$ and with $100 \mu \mathrm{L} / 40 \mathrm{~mL}$ Protease Inhibitor cocktail to preserve the protein stability. The hNaPRTase activity was tested using the assay previously reported [28] and resulted to be $0.27 \mu \mathrm{mol} / \mathrm{min} / \mathrm{mg}$. Using the same assay condition we tested the activity of human NaPRTase in presence of $100 \mu \mathrm{M}$ FK866 without observing any inhibition of enzyme activity. This procedure allowed us to obtain $20 \mathrm{mg}$ of pure and active human enzyme used for all crystallization trials.

\subsection{Crystallization and structure determination}

Crystals of hNaPRTase were obtained by means of the vapor diffusion technique in sitting drops. $4 \mu \mathrm{L}$ of a protein solution at a concentration of $15 \mathrm{mg} / \mathrm{mL}$, preincubated with $1 \mathrm{mM}$ Acetyl-CoA and $5 \mathrm{mM}$ DTT, were mixed with an equal volume of a reservoir solution containing $0.1 \mathrm{M}$ Sodium cacodylate $\mathrm{pH} 6.5$ and $1.7 \mathrm{M}$ Sodium acetate and equilibrated against $1.2 \mathrm{~mL}$ of the reservoir solution, at $4{ }^{\circ} \mathrm{C}$. Crystals grew to maximum dimension in about 5 days. For X-ray data collection, crystals were quickly equilibrated in a solution containing the crystallization buffer and $20 \%$ glycerol as the cryo-protectant and flash frozen at $100 \mathrm{~K}$ under a stream of liquid nitrogen. Data up to $2.9 \AA$ resolution were collected at the beamline ID23 EH1 of the European Synchrotron Radiation Facility (ESRF, Grenoble, France). Analysis of the diffraction data set collected allowed us to assign the crystal to the orthorhombic $\mathrm{P} 2{ }_{1} 2{ }_{1} 2_{1}$ space group with cell dimensions $a=88.0 \AA b=101.1 \AA$ and $c=125.1 \AA$ containing two molecules per asymmetric unit with a corresponding solvent content of $49 \%$. Data were processed using the program package XDS [32] and the CCP4 suite of program [33] were used for scaling. The structure determination of hNaPRTase was carried out by means of the molecular replacement technique using the coordinates of a monomer of Enterococcus faecalis NaPRTase as the search model (Protein Data Bank ID code 2F7F). Firstly, an improvement of the quality of the search model was carried out by using information contained in the sequence alignment between the hNaPRTase and the E. faecalis NaPRTase through the program SCULPTOR [34]. The new procedure Phenix.mr_rosetta [35], that combines crystallographic and structure-modeling algorithms from Phenix and Rosetta, respectively, was used to automatically determine the hNaPRTase structure. Phenix.mr_rosetta returned a unique molecular replacement solution with an LLG of 44.56 and TFZ =7.4. The initial model was subjected to iterative cycles of crystallographic refinement with the program REFMAC5 [36], alternated with manual graphic sessions for model building using the program Coot [37]. 5\% of randomly chosen reflections were excluded from refinement of the structure and used for the Free $\mathrm{R}$ factor calculation [38]. The program ARP/warp [39] was used for adding solvent molecules. Refinement was continued until convergence to $\mathrm{R}$ factor and Free $\mathrm{R}$ factor of 0.19 and 0.24 respectively with ideal geometry. Data collection and refinement statistics are given in Table 1.

\subsection{Molecular modeling}

A molecular dynamics simulation was performed using the GROMACS simulation package v.4.5.3 [40] with the standard

Table 1

Data collection and refinement statistics.

\begin{tabular}{ll}
\hline & hNaPRTase crystal \\
\hline Data collection & \\
Space group & $\mathrm{P} 2{ }_{1} 2_{1} 2_{1}$ \\
Cell dimensions & \\
$a, b, c(\AA)$ & $88.0,101.1,125.1$ \\
$\alpha, \beta, \gamma\left({ }^{\circ}\right)$ & $90,90,90$ \\
Resolution $(\AA)$ & 2.9 \\
$R_{\text {pim }} / R_{\text {merge }}$ & $6.1(20.5) / 11.4(37)$ \\
Mean(I)/sd $(\mathrm{I})$ & $10.0(3.7)$ \\
Completeness $(\%)$ & $99.7(99.3)$ \\
Redundancy & $4.4(4.2)$ \\
Refinement & \\
Resolution $(\AA)$ & 2.9 \\
No. reflections & $25,261(2464)$ \\
$R_{\text {work }} / R_{\text {free }}$ & $0.19 / 0.24$ \\
No. atoms & \\
Protein & 7576 \\
Water & 98 \\
Mean B-factors & \\
Protein $\left(\AA^{2}\right)$ & 42.67 \\
Water $\left(\AA^{2}\right)$ & 33.60 \\
R.m.s deviations & \\
Bond lengths $(\AA)$ & 0.014 \\
Bond angles $\left({ }^{\circ}\right)$ & 1.83 \\
\hline
\end{tabular}


GROMOS96 force field. The X-ray crystal structure of hNaPRTase was energy-minimized in vacuo, using 1000 steps of steepest descent in order to remove distorted geometries and with the protein embedded in a solvent box. The next step was a 2 ns simulation with position restraints to the protein, followed by a 500 ps unrestrained simulation. The last $500 \mathrm{ps}$ of the simulation were used to compute an average structure for hNaPRTase, which, after minimization with 1000 steps of steepest descent, represents the final model. After aminoacids stereochemistry has been assessed with the program PROCHECK [41], the model was used for docking simulations. The ligands tested for docking were FK866, Na, NaMN, Na plus PRPP and ATP. Ligand structures were built from a SMILES string and were minimized using Omega2 [42]. The docking simulations were performed using FRED, and using the default settings [43]. Each ligand-NaPRTase complex was subsequent subjected to a molecular structure optimization using SZYBKI [44] in order to refine the molecular structure using the Merck Molecular Force Field with solvent effect.

\subsection{Deposition}

The atomic coordinates and structure factors of human NaPRTase have been deposited with the Protein Data Bank (www.rcsb.org) with accession code: 4YUB.

\subsection{Illustrations}

Figures were generated by using the program pymol [45].

\section{Results and discussion}

\subsection{Overall quality of the model}

The three-dimensional structure of hNaPRTase has been solved by molecular replacement and refined at a resolution of $2.9 \AA$. The crystal asymmetric unit contains a dimer of hNaPRTase and a total of 98 solvent molecules. The two protein molecules have been designated as monomers A and B (Fig. 2). Monomer A contains 501 residues out of 538 and no electron density is present for the following regions: first 15 residues at the $\mathrm{N}$-terminus, residues 379-389, residues 409-415, and last five residues at the C-terminus. Monomer B contains 501 residues out of 538 and no electron density is present for the following regions: first 15 residues at the N-terminus, residues 379-389, residues 408-414, and last four residues at the C-terminus. The stereochemistry of the model has been assessed with the program PROCHECK [41]: $99 \%$ of protein residues fall in the favoured regions of the Ramachandran plot with no outliers.

\subsection{Overall structure of human NaPRTase monomer}

Human NaPRTase monomer folds into $17 \alpha$-helices, 24 $\beta$-strands and the connecting loops organized in two domains: a first domain characterized by an irregular $\alpha / \beta$ barrel and a second open-faced sandwich domain (Fig. 3). In particular, as shown in the protein topology diagram in Fig. 3, the open-face sandwich domain is composed of residues provided by both the $\mathrm{N}$ - and C-terminal regions of the protein (residues 16-127 and 390-534). It consists of $4 \alpha$-helices disposed like a shield and delimiting the open space where $15 \beta$-strands form 2 antiparallel $\beta$-sheets, showing a specular two-by-two arrangement. Indeed, the seven-stranded fully antiparallel $\beta$-sheet $(\beta 1, \beta 3, \beta 5, \beta 15, \beta 16, \beta 22$ and $\beta 23)$ and the four-stranded partially antiparallel $\beta$-sheet $(\beta 17, \beta 18, \beta 21$ and $\beta 24)$ face each other. The other two smaller $\beta$-sheets ( $\beta 2, \beta 4$ and $\beta 19, \beta 20)$ delimit the domain. The longest helix $\alpha 5$, with 8 turns (residues 128-159) connects the two domains. The irregular $\alpha / \beta$ barrel domain (residues $160-378$ ) contains a six-stranded $\alpha / \beta$ core ( $\beta 6, \beta 7, \beta 11, \beta 12, \beta 13$, and $\alpha 6, \alpha 11, \alpha 12, \alpha 13, \alpha 14$ ) that identifies hNaPRTase as a member of the type II Phosphoribosyltransferases (PRTases) subfamily. The two monomers present in the asymmetric unit have the same conformation showing a r.m.s. deviation of $0.5 \AA$ after superposition based on all $\mathrm{C} \alpha$ atoms. The hNaPRTase structural organization turns out to be highly similar to that of $E$. faecalis NaPRTase, used as a model for the molecular replacement. Indeed, the two structures can be optimally superimposed with a r.m.s.d. of $2.4 \AA$ based on 482 equivalent $\mathrm{C} \alpha$ atoms. As could be expected, the overall fold is highly conserved with two domains connected by a long $\alpha$-helix. Moreover, in spite of the low sequence identity (34\%) the active site residues are conserved in the two enzymes revealing an identical mode of binding for substrates. In particular, Y17, H205, R262, S206 K333 R163 and T162 that in E. faecalis NaPRTase defines the enzyme active site are strictly conserved in hNaPRTase with equivalent positions occupied by Y21, H213, R318, S214 K396 R171 and L170. The high degree of structural conservation observed between the human and the bacterium NaPRTases could be due to an evolutionary adaptation. Indeed, E. faecalis is a commensal bacterium inhabiting the gastrointestinal tracts of humans where, in the small intestine, $\mathrm{Na}$ is a preferred source for NAD synthesis.

\subsection{The dimer of human NaPRTase}

hNaPRTase has a predicted molecular weight of $58 \mathrm{KDa}$ and it has been reported to show a native molecular mass of about $87,000 \mathrm{Da}$ by gel filtration [46,28]. The crystal structure of hNaPRTase reveals the presence of an intimately associated dimer in the asymmetric unit with $3000 \AA^{2}$ of the surface area buried at the dimer interface. The two monomers are arranged head to tail with the N-terminal domain in one monomer contacting the $\alpha / \beta$ barrel in the other monomer (Fig. 2). Therefore our structural data confirm that the minimal functional unit in the hNaPRTase enzyme consists of a dimer as already proposed for Thermoplasma acidophilum NaPRTase [47]. Moreover, the major interactions established at the dimeric interface in TaNaPRTase were observed to be of ionic rather than hydrophobic nature [47]. Our structural analysis confirmed that the main interactions between monomers are of ionic nature also in hNaPRTase.

\subsection{The active site}

Since we have been unable to obtain crystals of hNaPRTase in complex with any ligand, the binding mode of substrate/product to the enzyme active site was investigated through molecular docking simulations. The functional dimeric model of hNaPRTase was first re-built using the X-ray crystal structure and subsequently refined by molecular dynamics simulations. Na, NaMN, Na plus PRPP, and ATP were docked in the generated model. As already reported in the previously characterized NaPRTases [47], the active site consists of a pocket located at the dimer interface. In particular, in hNaPRTase it is delimited by the loops connecting $\alpha$-helix 6 with $\beta$-strand 6 and $\alpha$-helix 8 with $\beta$-strand 8 of $\alpha / \beta$ barrel domain of monomer $A$ and completed by residues of $\beta$-strand 11 and $\alpha$-helix 1 from monomer B (Fig. 4). According to our modeling procedure the pyridine ring of $\mathrm{Na}$ establishes contacts with the protein environment that involve a $\pi-\pi$ stacking interaction with Y21B and Van der Walls contacts with L170A, G209A and L211A. In addition, the carboxyl group of $\mathrm{Na}$ is engaged in a strong ionic interaction with R318A (Fig. 5a). It has previously been reported that the G201 to Ala substitution causes a 90\% reduction of enzyme activity even in the presence of ATP and that the Y21 to Ala substitution results in the complete loss of activity in absence 

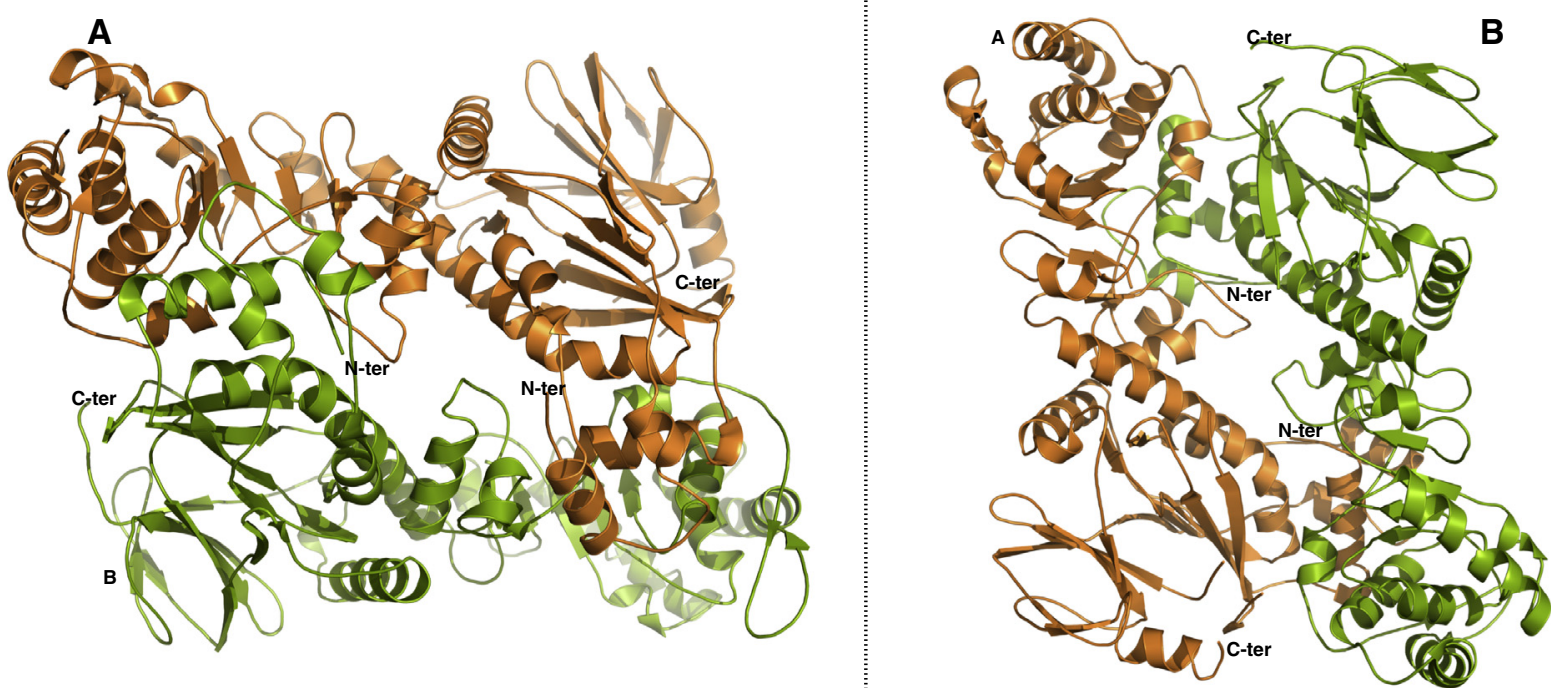

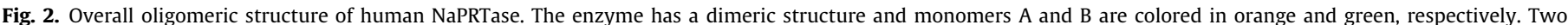
different orientations of the dimer, showing the head-to-tail arrangement of the monomers, are represented. N-ter, N-terminus; C-ter, C-terminus.

of ATP and in a 50\% reduction in the presence of ATP [28]. Our data are therefore in agreement with the functional analysis carried out by site directed mutagenesis and provide a solid structural explanation for the role played by these residues in catalysis. We then investigated the mode of binding for Na and PRPP when simultaneously bound to the enzyme active site (Fig. 5b). PRPP appears to be stabilized by two ionic interactions with R171A and K396B that are engaged in a salt bridge with the phosphate and pyrophosphate moieties, respectively. PRPP is further stabilized by two hydrogen bonds established between its ribose oxygen and R318A and between the pyrophosphate moiety and S214A. Our observations confirm the functional analysis carried out by site-directed mutagenesis previously reported in which the replacement of R318 with Ala completely abolish the enzyme activity in absence of ATP while a reduction of $50 \%$ is observed in the presence of ATP [28]. The ATP binding mode was also investigated and turned out to be quite similar to that observed for PRPP (Fig. 5d). In particular the ATP $\gamma$ phosphate group is salt bridged to $\mathrm{K} 396 \mathrm{~B}$, one of the key residue for PRPP binding, while the $\beta$ phosphate group is involved in hydrogen bonds with H213A (backbone amide) and S214A. The adenine moiety of ATP is involved in a $\pi$-polar interaction with $\mathrm{R} 318 \mathrm{~A}$. Also in this case, our observations remark an important role in catalysis for $\mathrm{H} 213$ that when mutated to Ala was reported to cause a $50 \%$ reduction of the enzyme activity [28]. Finally, the binding mode of the product NaMN was investigated (Fig. 5c). As expected, the orientation of both the $\mathrm{Na}$ and phosphate moieties in the mononucleotide, is identical to what observed for the corresponding chemical entities in the model of the Na-PRPP enzyme complex. In conclusion, our structural models for substrate binding provide a solid explanation for the role played in catalysis by residues that were previously reported to be essential for enzyme activity by a functional analysis based on site directed mutagenesis [28]. The perfect coherence between biochemical data reported in previous study and our structural observations, also proves that our molecular docking simulation are robust. Therefore, besides R318, Y21, H213 that were already subjected to site directed mutagenesis and that we confirm to be essential residues for catalysis, we propose that R171, K396 and S214 also play an essential role in substrate recognition. Moreover, we identify R318 as the major player in catalysis. Indeed, this residue is involved in the binding of all substrates by recognizing different chemical entities: the carboxylic moiety of $\mathrm{Na}$, the ribose ring of PRPP and the adenine base of ATP. In addition, our in silico model of the complex hNaPRT-Na and hNaPRT-NaMN allows a detailed structural comparison of the mode of binding of $\mathrm{Na}, \mathrm{Nam}$ and QA in the three phosphoribosyltransferases. At the level of the active sites we observe a high degree of structural conservation mainly between hNaPRTase and hNMPRTase, while a less significant conservation is observed with hQAPRTase. However, the mode of binding of all the three different pyridines is conserved, while different is the protein milieu surrounding the substrate. In particular, hQAPRTase provides a more positively charged environment that is required for the recognition of QA that carries two negatively charged carboxylic groups. Although our structural analysis does not disclose any obvious common features for the catalytic mechanism adopted by hQAPRTase, hNMPRTase and hNaPRTase, a conserved mode of binding of the pyridine ring containing substrate clearly emerges. More information can be extracted if the structural analysis is confined to hNaPRTase and hNMPRTase. In these two enzymes, the mode of binding of $\mathrm{Na}$ and Nam appears highly similar. Indeed, the pyridine ring is sandwiched by an aromatic/hydrophobic clamp in both enzymes: Y18/F193 in hNMPRTase and Y21/L170 in hNaPRTase. R311, R196 and K400 that in hNMPRTase play a key role in the binding of PRPP, are strictly conserved in hNaPRTase (R318, R171 and K396). On the other hand, a specific residue appears to participate in guaranteeing the strikingly substrate specificity displayed by hNMPRTase and hNAPRTase for nicotinamide versus nicotinic acid. While in hNMPRTase D219 interacts with the amide moiety of Nam, in hNaPRTase the structurally equivalent position is occupied by S214 contributing to direct the enzyme specificity toward nicotinate. Other structural determinants that do not clearly emerge in our docking models are however required to fully explain the strict specificity toward nicotinic acid displayed by hNaPRTase. Overall, a conserved mode of binding of QA, Na and Nam in the respective phosphoribosyltransferase is observed. However, the structural architecture of the active site is sensibly different in hQAPRTase and peculiar traits also feature hNMPRTase and hNaPRTase. Therefore, we propose 


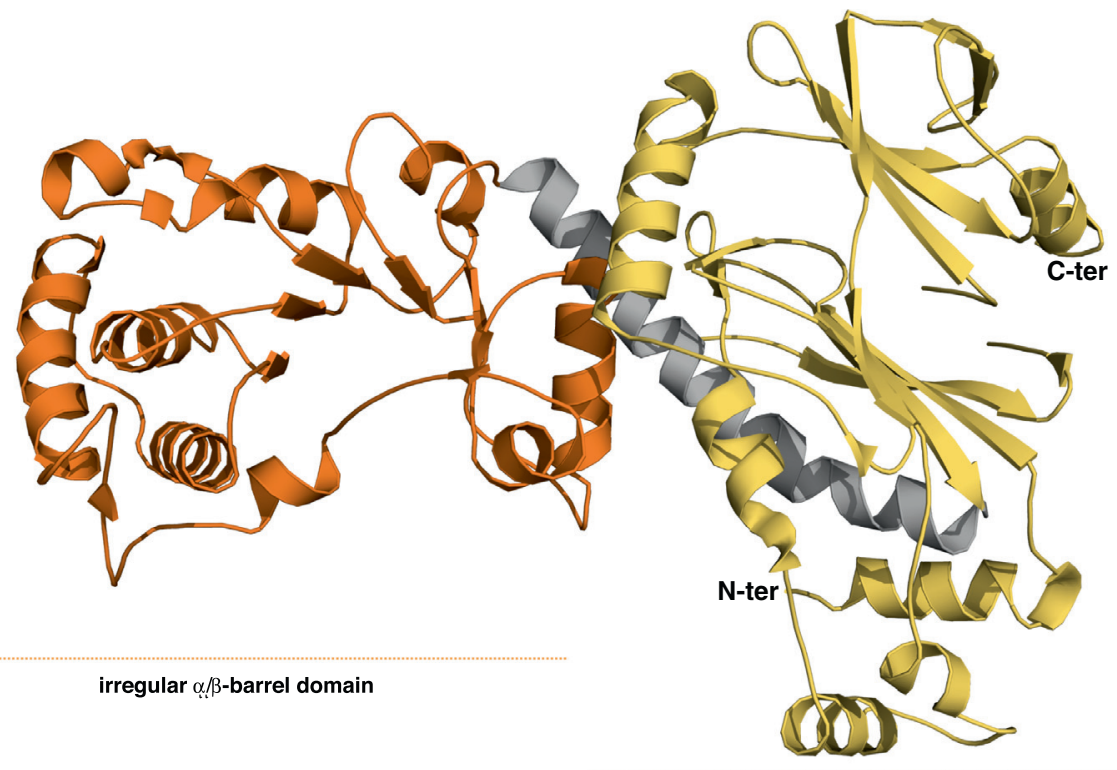

open-faced sandwich domain

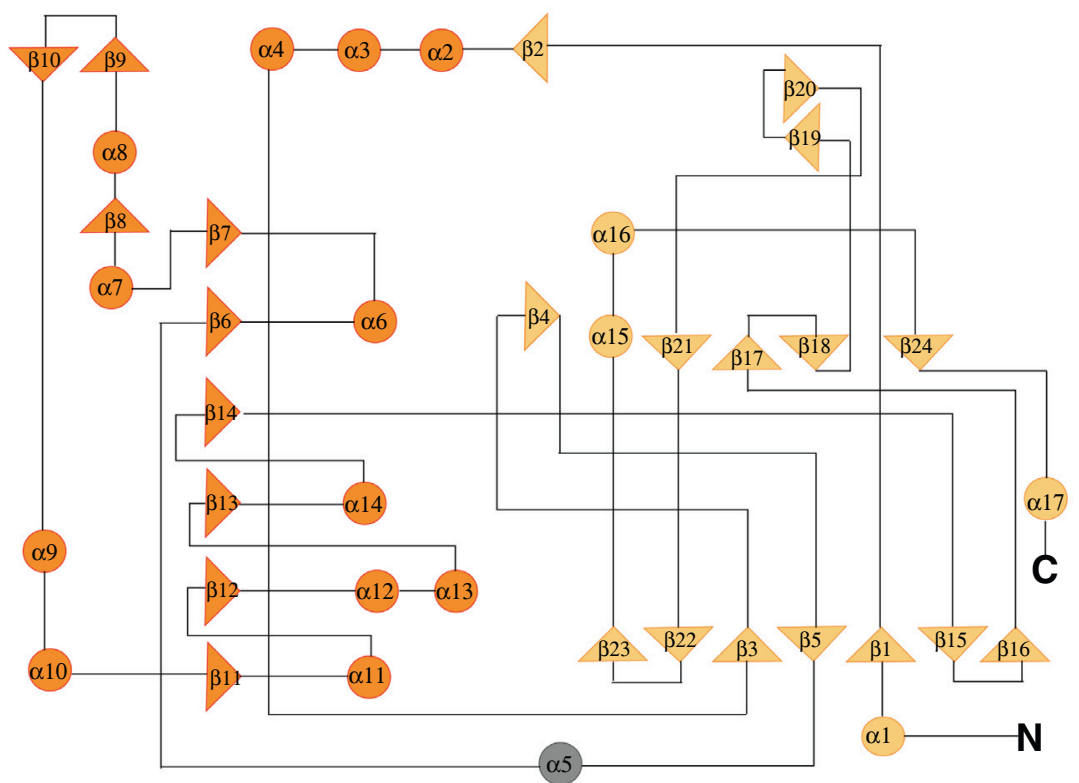

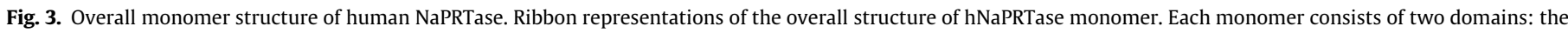

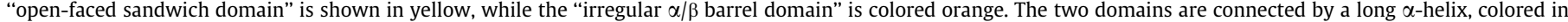

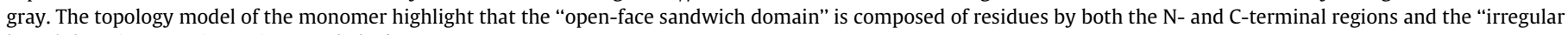
barrel domain" contains a six-stranded $\alpha / \beta$ core.

that the wealth of structural information that is now available for all the three enzymes can be successfully exploited for the design of highly selective inhibitors.

\subsection{Comparison with hNMPRTase and hQAPRTase}

Despite sharing very limited sequence similarity, hNaPRTase shows a molecular fold that closely resembles those firstly described for hNMPRTase [31,48] and hQAPRTase [49] (Fig. 6). This fact is in accordance with their common function, consisting in the transfer of the phosphoribosyl group from PRPP onto their respective substrates. A DALI search shows a significant similarity with hNMPRTase with a $Z$-value of 22.2 and a r.m.s. deviations of $3.6 \AA$ based on superposition of 463 equivalent $\mathrm{C} \alpha$ atoms.
hQAPRTase is the smallest in size among the three PPRTases, in agreement with the hypothesis that it is the most ancient [50]. At the contrary, hNaPRTase contains 46 and 241 more amino acid residues than hNMPRTase and hQAPRTase, respectively. The structural superposition of the three enzymes highlights how some of the additional residues in hNaPRTase form two structural regions that, from a Blastp search, appear to be univocally present in mammal NaPRTases (Fig. 6). The first unique region spans amino acids 223-264 and contains two $\beta$-strands $(\beta 9-\beta 10)$ and one $\alpha$-helix ( $\alpha 9$ ) that extend the borders of the $\alpha / \beta$ barrel domain and contribute to contact the other monomer (Fig. 3). Indeed, the side chain of D227 is hydrogen bonded to the side chain of R31 of the opposite monomer. The second unique region (447-472) of hNaPRTase encompasses three $\beta$-strands $(\beta 19, \beta 20$ and $\beta 21)$ that 


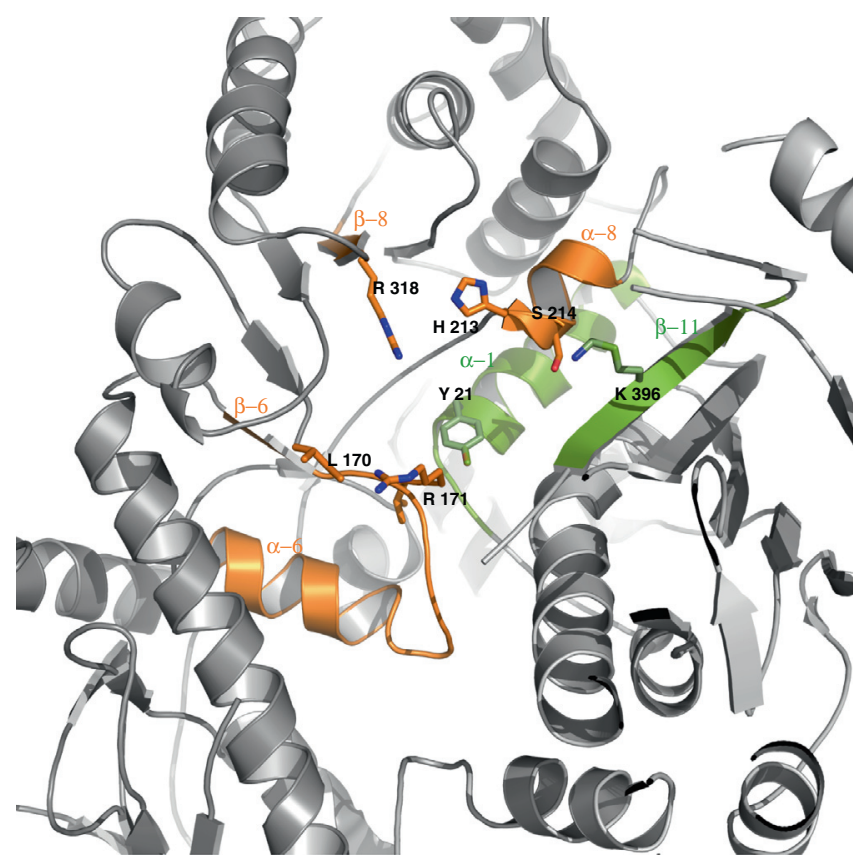

Fig. 4. Active site representation of human NaPRTase. Ribbon representation of the active site formed at the dimeric interface, between the $\alpha / \beta$ barrel domain of monomer A, colored in orange, and the monomer B, shown in green. Side chains of key residues involved in catalysis are shown.

stand at the bottom of the open-faced sandwich (Fig. 3). These two peculiar regions in hNaPRT are located on the enzyme surface and do not have any structural impact on the enzyme active site.
Therefore, there is no evidence of their involvement in catalysis or substrate binding. On the other hand, since the activity of extracellular hNMPRTase as a potent cytokine is well documented [51], we speculate that the structural variation of the protein surface may sustain still unknown functions of an extracellular form of hNaPRTase. The analysis of conserved versus peculiar traits in the active site of the three human phosphoribosyltransferases has been carried out based on optimal structural superposition. The active site of hNAPRTase and hNMPRTase shows a high degree of structural conservation that appears necessary for the binding of the common substrate PRPP and the highly similar substrates Na and Nam. Indeed, for the latter case, the pyridine ring recognition is guaranteed by few strictly conserved residues; in particular, Y21, H213, R318 in hNaPRTase appear to be structurally equivalent to Y18, H247 and R311 in hNMPRTase; on the other hand, F193 that in hNMPRTase provides an aromatic stacking interaction with the Nam pyridine ring is substituted by L170 in hNaPRTase. Of note is the observed different conformation of another strictly conserved residue located in the enzyme active site, i.e. R196 in hNMPRTase that is equivalent to R171 in hNaPRTase. In the latter, the positively charged side chain closes up the enzyme active site and is one of the structural determinants hampering binding of FK866 to hNaPRTase. When we included in the structural analysis hQAPRTase, a lower degree of structural conservation amongst all the three enzymes, emerged. First, the active site in hQAPRTase is sensibly more positively charged with residues R102, R138, R161, K139 and K171 all involved in the binding of QA that carries two carboxylic groups. Among all these residues R102 appears to be strictly structurally conserved in all the three phosphoribosyltransferase occupying the equivalent position of R196 in hNAMPTase and R171 in hNaPRTase. This residue interacts with the functional moiety present in position 3 of the pyridine ring in all the three
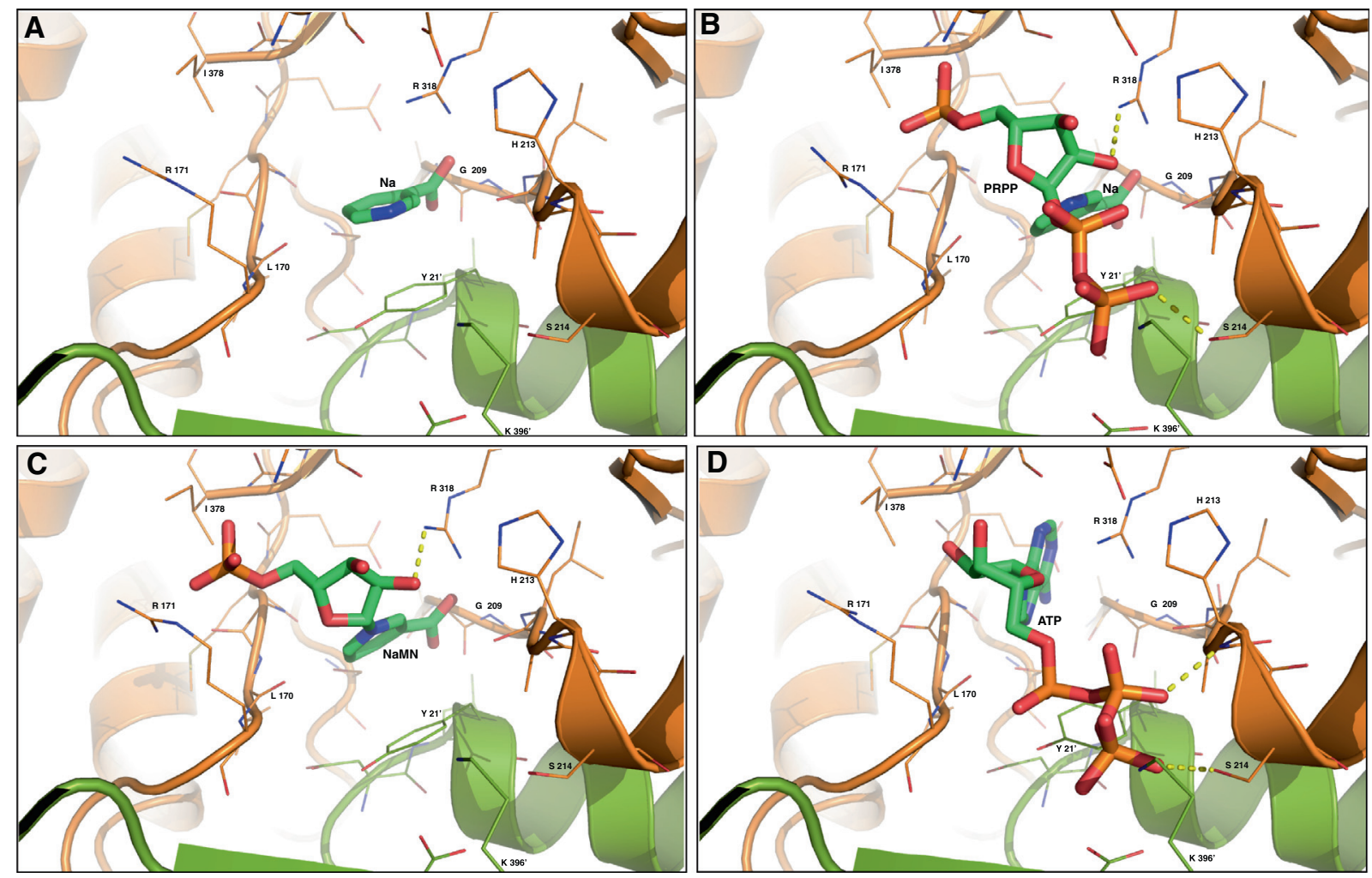

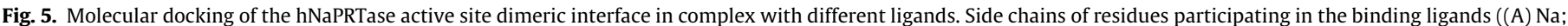

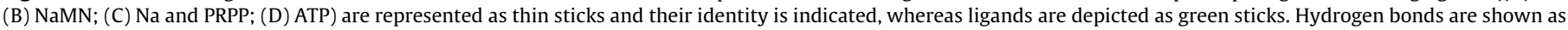
yellow dotted lines. 


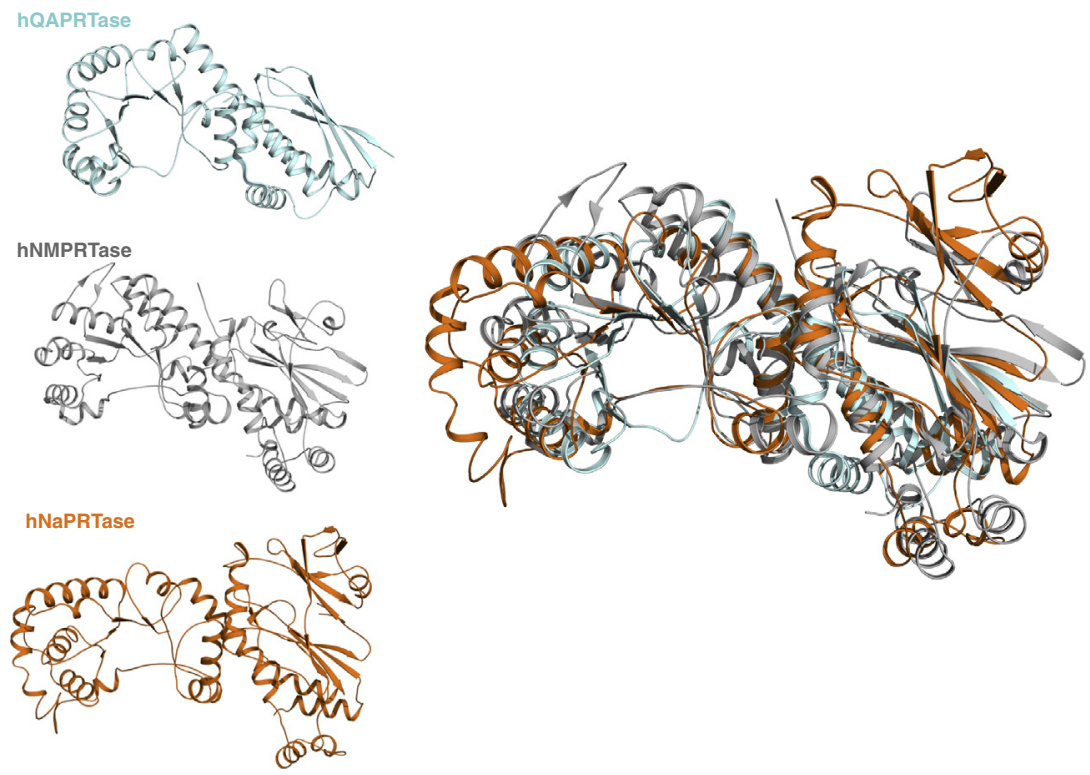

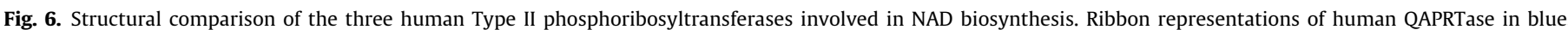
NMPRTase in gray and NaPRTase in orange are shown separately (left panel). The structural superposition of the three enzymes is also represented (right panel).
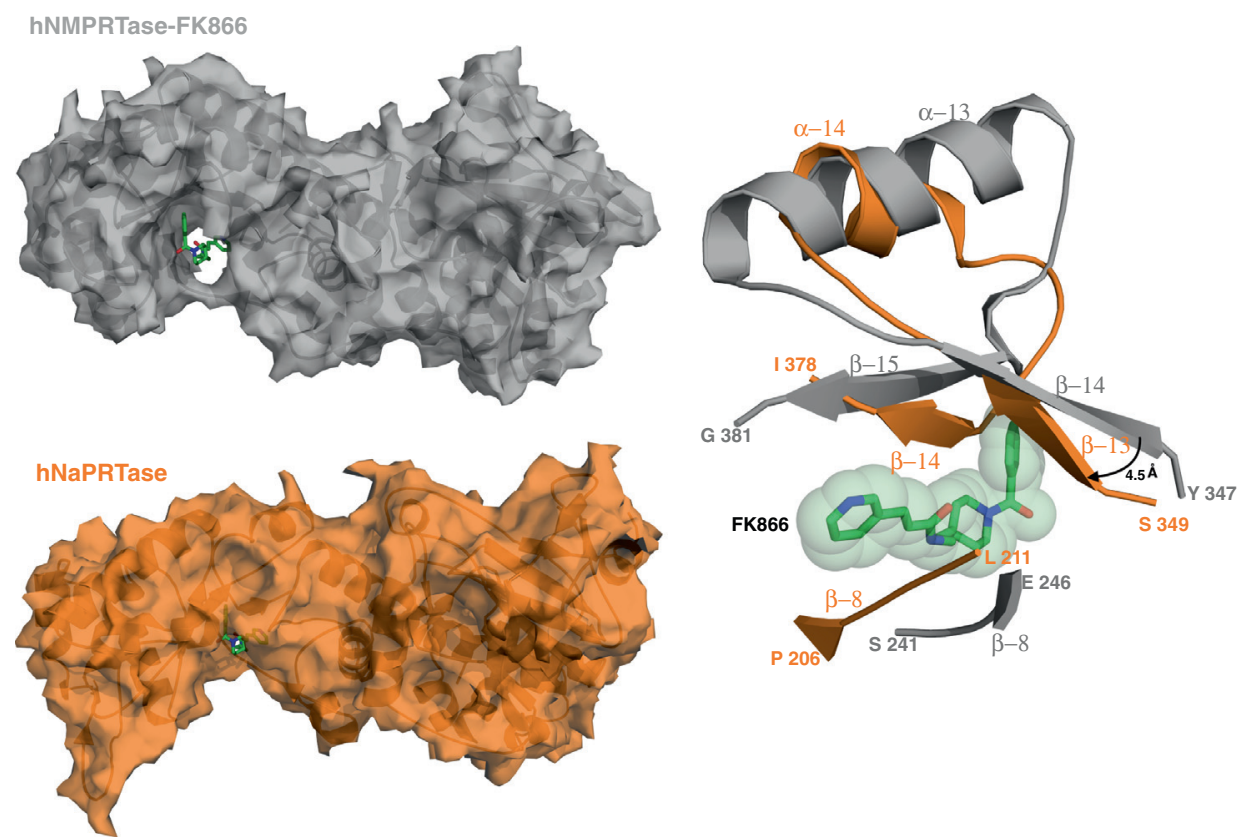

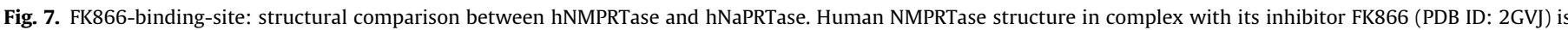

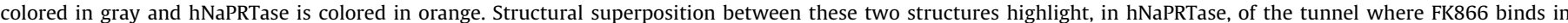

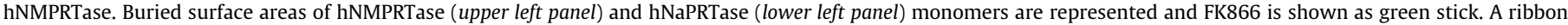

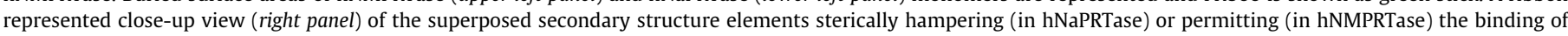
FK866. Steric hindrance of FK866 is represented by light green spheres.

different ligands: QA, Na and Nam. On the other hand, R138 and R161 in hQAPRTase appear as a peculiar feature of this enzyme and interact with the second carboxyl group present only in the structure of QA, in position 2 of its pyridine ring.

\subsection{Lack of inhibition by FK866}

FK866 is a potent hNMPRTase competitive inhibitor endowed with a strong antitumor activity and is currently undergoing clinical trials for the treatment of cancer [31]. Surprisingly, FK866 does not inhibit hNaPRTase [31] and an explanation of this unexpected observation is still missing. The inhibitory mechanism of action on the target has been investigated by structural studies [31]. FK866 binds to hNMPRTase in a tunnel observed at the dimer interface and defined by the parallel $\beta$ sheet of the $\alpha / \beta$ barrel domain of each monomer [31]. In particular, two small $\beta$ strands ( $\beta 14$ and $\beta 15$ ) that participate in shaping the tunnel and are located in close proximity of the enzyme's active site, contribute to the recognition and binding of FK866. In hNaPRTase the corresponding region ( $\beta 13$ and $\beta 14$ ) revealed a different orientation determined by a 10 residues 
deletion that shorten the preceding alpha helix ( $\alpha 14)$. The resulting structural arrangement sterically prevents the binding of FK866 to hNaPRTase due to severe clashes between the inhibitor and the protein region defining the tunnel (Fig. 7).

\section{Conclusion}

NaPRTase is the first enzyme in the Preiss-Handler NAD salvage pathway and therefore has an important role in NAD metabolism, which is involved in a large number of physiological and pathological conditions in all organisms. Beyond its role in NAD biosynthesis, it was hypothesized that, in humans, NaPRTase could be linked to other central metabolic pathways [27] as suggested also for hACMSD, an enzyme regulating the novo NAD synthesis [52]. Several studies have underlined the NaPRTase importance for NAD homeostasis in mammals, but no crystallographic data are available for higher eukaryotes. Our investigations reveal a high degree of structural conservation at the level of the overall fold with both bacterial NaPRTase and hNMPRTase. However, while in the case of the bacterial enzyme the structural similarity extend also to the active site, significant differences emerge in such a region with hNMPRTase. Indeed, we confirm that the functional unit of hNaPRTase is a dimer with the active site located at the domains interface in each monomer, as observed in hNMPRTase. On the other hand, the structural organization of the active site in the two enzymes, clearly shows peculiar traits that explain both the strikingly different substrate specificity as well as the specific inhibitory action of FK866 on hNMPRTase. hNaPRT is a medically relevant enzyme; its expression levels have been shown to vary consistently among populations and in different pathological conditions [53-56] including cancer where variants of the enzyme have also been identified [54]. Moreover, it has recently been reported that the adverse effects caused by reactive oxygen species induced by the treatment with the anticancer hNMPRTase inhibitors (FK866 and analogues), can be reduced by a co-treatment with nicotinic acid that is linked to the over-expression of hNaPRTases [57]. Indeed, the administration of nicotinic acid protected non-tumor cells from toxic effects resulting from depletion of NAD caused by hNMPRTase inhibition [57]. Therefore, a potential future effective antitumor therapy targeting NAD biosynthesis should be featured by both a strong blockage of hNMPRTase to obtain high toxicity toward tumor cells and no inhibition of hNaPRTase in order to reduce the toxic effect of the anticancer drug on non-tumoral cells. The availability of the crystal structure of both hNMPRTase and hNaPRTase is of high relevance for the structure-based rational design of potent and selective, i.e. acting on hNMPRTase only, inhibitors of potential medical relevance. In conclusion our structural data may find application for cancer treatment and may help in elucidating the complex and yet unclear interconnections among distinct metabolic pathways in humans.

\section{Contributions}

S.G., A.S.M., G.O., A.M. and G.M. performed experiments and collected and analyzed the data; S.G. designed the research; S.G. and M.R. wrote the paper.

\section{Competing financial interests}

The authors declare no competing financial interests.

\section{Acknowledgments}

The authors would like to thank Professor Nadia Raffaelli (Università Politecnica delle Marche, Italy) for critical reading of the manuscript and helpful discussion. This work was supported by the University of Piemonte Orientale (Grant Ricerca di Ateneo).

\section{References}

[1] Belenky, P., Bogan, K.L. and Brenner, C. (2007) NAD+ metabolism in health and disease. Trends Biochem. Sci. 32, 12-19.

[2] Magni, G., Amici, A., Emanuelli, M., Orsomando, G., Raffaelli, N., et al. (2004) Enzymology of NAD+ homeostasis in man. Cell. Mol. Life Sci. 61, 19-34.

[3] Berger, F., Ramirez-Hernandez, M.H. and Ziegler, M. (2004) The new life of a centenarian: signalling functions of NAD(P). Trends Biochem. Sci. 29, 111-118.

[4] Ziegler, M. (2000) New functions of a long-known molecule. Emerging roles of NAD in cellular signaling. Eur. J. Biochem. 267, 1550-1564.

[5] Guse, A.H. (2005) Second messenger function and the structure-activity relationship of cyclic adenosine diphosphoribose (cADPR). FEBS J. 272, 45904597.

[6] Marmorstein, R. (2004) Structure and chemistry of the Sir2 family of NAD+dependent histone/protein deacetylases. Biochem. Soc. Trans. 32, 904-909.

[7] Magni, G., Orsomando, G., Raffelli, N. and Ruggieri, S. (2008) Enzymology of mammalian NAD metabolism in health and disease. Front. Biosci. 13, 61356154.

[8] Araki, T., Sasaki, Y. and Milbrandt, J. (2004) Increased nuclear NAD biosynthesis and SIRT1 activation prevent axonal degeneration. Science 305, 1010-1013.

[9] Di Stefano, M. and Conforti, L. (2013) Diversification of NAD biological role: the importance of location. FEBS J. 280, 4711-4728.

[10] Guarente, L. and Picard, F. (2005) Calorie restriction - the SIR2 connection. Cell 120, 473-482.

[11] Bieganowski, P. and Brenner, C. (2004) Discoveries of nicotinamide riboside as a nutrient and conserved NRK genes establish a Preiss-Handler independent route to NAD+ in fungi and humans. Cell 117, 495-502.

[12] Magni, G., Amici, A., Emanuelli, M., Raffaelli, N. and Ruggieri, S. (1999) Enzymology of NAD+ synthesis. Adv. Enzymol. Relat. Areas Mol. Biol. 73, 135182.

[13] Garavaglia, S., D’Angelo, I., Emanuelli, M., Carnevali, F., Pierella, F., et al. (2002) Structure of human NMN adenylyltransferase. A key nuclear enzyme for NAD homeostasis. J. Biol. Chem. 277, 8524-8530.

[14] Zhai, R.G., Rizzi, M. and Garavaglia, S. (2009) Nicotinamide/nicotinic acid mononucleotide adenylyltransferase, new insights into an ancient enzyme. Cell. Mol. Life Sci. 66 (17), 2805-2818.

[15] Rizzi, M., Bolognesi, M. and Coda, A. (1998) A novel deamido-NAD+-binding site revealed by the trapped NAD-adenylate intermediate in the NAD+ synthetase structure. Structure 6 (9), 1129-1140.

[16] Revollo, J.R., Grimm, A.A. and Imai, S. (2004) The NAD biosynthesis pathway mediated by nicotinamide phosphoribosyltransferase regulates Sir2 activity in mammalian cells. J. Biol. Chem. 279, 50754-50763.

[17] Rongvaux, A., Andris, F., Van Gool, F. and Leo, O. (2003) Reconstructing eukaryotic NAD metabolism. BioEssays 25, 683-690.

[18] Mori, V.A.A., Mazzola, F., Di Stefano, M., Conforti, L., Magni, G., Ruggieri, S., Raffaelli, N. and Orsomando, G. (2014) Metabolic profiling of alternative NAD biosynthetic routes in mouse tissues. PloSOne 9 (11), 1-27.

[19] Jackson, T.M., Rawling, J.M., Roebuck, B.D. and Kirkland, J.B. (1995) Large supplements of nicotinic acid and nicotinamide increase tissue NAD+ and poly(ADP-ribose) levels but do not affect diethylnitrosamine-induced altered hepatic foci in Fischer-344 rats. J. Nutr. 125, 1455-1461.

[20] Handler, P. and Kohn, H.I. (1943) The mechanisms of cozymase synthesis in the human erythrocyte: a comparison of the roles of nicotinic acid and nicotinamide. J. Biol. Chem. 150, 447-452.

[21] Henderson, L.M. (1983) Niacin. Annu. Rev. Nutr. 3, 289-307.

[22] Hara, N., Yamada, K., Shibata, T., Osago, H., Hashimoto, T., et al. (2007) Elevation of cellular NAD levels by nicotinic acid and involvement of nicotinic acid phosphoribosyltransferase in human cells. J. Biol. Chem. 282, 2457424582.

[23] Jacobson, E.L., Kim, H., Kim, M., Williams, J.D., Coyle, D.L., et al. (2007) A topical lipophilic niacin derivative increases NAD, epidermal differentiation and barrier function in photodamaged skin. Exp. Dermatol. 16, 490-499.

[24] Bodor, E.T. and Offermanns, S. (2008) Nicotinic acid: an old drug with a promising future. Br. J. Pharmacol. 153 (Suppl. 1), 68-75.

[25] Wise, A., Foord, S.M., Fraser, N.J., Barnes, A.A., Elshourbagy, N., et al. (2003) Molecular identification of high and low affinity receptors for nicotinic acid. J. Biol. Chem. 278, 9869-9874.

[26] Soga, T., Kamohara, M., Takasaki, J., Matsumoto, S., Saito, T., et al. (2003) Molecular identification of nicotinic acid receptor. Biochem. Biophys. Res. Commun. 303, 364-369.

[27] Zamporlini, F., Ruggieri, S., Mazzola, F., Amici, A., Orsomando, G. and Raffaelli, N. (2014) Novel assay for simultaneous measurement of pyridine mononucleotides synthesizing activities allows dissection of the NAD biosynthetic machinery in mammalian cells. FEBS J. 281 (22), 5104-5119.

[28] Galassi, L., Di Stefano, M., Brunetti, L., Orsomando, G., Amici, A., et al. (2012) Characterization of human nicotinate phosphoribosyltransferase: kinetic studies, structure prediction and functional analysis by site-directed mutagenesis. Biochimie 94, 300-309.

[29] Gross, J., Rajavel, M., Segura, E. and Grubmeyer, C. (1996) Energy coupling in Salmonella typhimurium nicotinic acid phosphoribosyltransferase: 
identification of His-219 as site of phosphorylation. Biochemistry 35, 3917 3924.

[30] Kosaka, A., Spivey, H.O. and Gholson, R.K. (1971) Nicotinate phosphoribosyltransferase of yeast. Purification and properties. J. Biol. Chem. 246, 3277-3283.

[31] Khan, J.A., Tao, X. and Tong, L. (2006) Molecular basis for the inhibition of human NMPRTase, a novel target for anticancer agents. Nat. Struct. Mol. Biol. $13,582-588$

[32] Kabsch, W. (2010) XDS. Acta Crystallogr. D Biol. Crystallogr. 66 (Pt 2), 125132.

[33] Collaborative Computational Project, Number 4 (1994) The CCP4 suite: programs for protein crystallography. Acta Crystallogr. D Biol. Crystallogr. 50 (5), 760-763.

[34] Bunkóczi, G. and Read, R.J. (2011) Improvement of molecular-replacement models with Sculptor. Acta Crystallogr. D Biol. Crystallogr. 67 (P4), 303-312.

[35] Terwilliger, T.C., Dimaio, F., Read, R.J., Baker, D., Bunkóczi, G., Adams, P.D., Grosse-Kunstleve, R.W., Afonine, P.V. and Echols, N. (2012) Phenix.mr_rosetta: molecular replacement and model rebuilding with Phenix and Rosetta. J. Struct. Funct. Genomics 13 (2), 81-90.

[36] Murshudov, G.N., Vagin, A.A. and Dodson, E.J. (1997) Refinement of macromolecular structures by the maximum-likelihood method. Acta Crystallogr. D53, 240-255.

[37] Emsley, P., Lohkamp, B., Scott, W.G. and Cowtan, K. (2010) Features and development of Coot. Acta Crystallogr. D Biol. Crystallogr. 66 (4), 486-501.

[38] Brunger, A.T. (1992) Free R value: a novel statistic quantity for assessing the accuracy of crystal structures. Nature 355 (6359), 472-475.

[39] Perrakis, A., Harkiolaki, M., Wilson, K.S. and Lamzin, V.S. (2001) ARP/wARP and molecular replacement. Acta Crystallogr. D Biol. Crystallogr. 57 (10), 14451450.

[40] Berendsen, H.J., Spoel, V.D. and Drunen, R.V. (1995) GROMACS: a messagepassing parallel molecular dynamics implementation. Comput. Phys. Commun. 95, 43-56.

[41] Laskowsky, R.A., Moss, D.S. and Thornton, J.M. (1993) Main-chain bond lengths and bond angles in protein structures. J. Mol. Biol. 231 (4), 1049-1067.

[42] N.M. Santa Fe, OpenEye Scientific Software, <http://www.eyesopen.com>. (b) Hawkins, P.C.D., Skillman, A.G., Warren, G.L., Ellingson, B.A. and Stahl, M.T. (2010) Conformer generation with OMEGA: algorithm and validation using high quality structures from the Protein Databank and Cambridge Structural Database. J. Chem. Inf. Model. 50, 572-584.

(c) Hawkins, P.C.D. and Nicholls, A. (2012) Conformer generation with OMEGA: learning from the data set and the analysis of failures. J. Chem. Inf. Model. 52, 2919-2936.

[43] N.M. Santa Fe, FRED, version 3.0.0; OpenEye Scientific Software, <http://www. eyesopen.com>.

(b) McGann, M. (2011) FRED pose prediction and virtual screening accuracy. J. Chem. Inf. Model. 51, 578-596.

[44] N.M. Santa Fe, SZYBKI, version 1.7.0; OpenEye Scientific Software, <http:// www.eyesopen.com>.

[45] 45Schrödinger LLC, The PyMOL Molecular Graphics System, version 1.3, 2010.
[46] Niedel, J. and Dietrich, L.S. (1973) Nicotinate phosphoribosyltransferase of human erythrocytes. Purification and properties. J. Biol. Chem. 248 (10), 3500

[47] Shin, D.H., Oganesyan, N., Jancarik, J., Yokota, H., Kim, R. and Kim, S.H. (2005) Crystal structure of a nicotinate phosphoribosyltransferase from Thermoplasma acidophilum. J. Biol. Chem. 280 (18), 18326-18335.

[48] Wang, T., Zhang, X., Bheda, P., Revollo, J.R., Imai, S. and Wolberger, C. (2006) Structure of Nampt/PBEF/visfatin, a mammalian NAD+ biosynthetic enzyme Nat. Struct. Mol. Biol. 13 (7), 661-662.

[49] Liu, H., Woznica, K., Catton, G., Crawford, A., Botting, N. and Naismith, J.H. (2007) Structural and kinetic characterization of quinolinate phosphoribosyltransferase (hQPRTase) from homo sapiens. J. Mol. Biol. 373 (3), 755-763.

[50] Chappie, J.S., Cànaves, J.M., Han, G.W., Rife, C.L., Xu, Q. and Stevens, R.C. (2005) The structure of a eukaryotic nicotinic acid phosphoribosyltransferase reveals structural heterogeneity among type II PRTases. Structure 13 (9), 1385-1396.

[51] Audrito, V., Serra, S., Brusa, D., Mazzola, F., Arruga, F., Vaisitti, T., Coscia, M. Maffei, R., Rossi, D., Wang, T., Inghirami, G., Rizzi, M., Gaidano, G., Garcia, J.G., Wolberger, C., Raffaelli, N. and Deaglio, S. (2015) Extracellular nicotinamide phosphoribosyltransferase (NAMPT) promotes M2 macrophage polarization in chronic lymphocytic leukemia. Blood 125 (1), 111-123, http://dx.doi.org/ 10.1182/blood-2014-07-589069.

[52] Garavaglia, S., Perozzi, S., Galeazzi, L., Raffaelli, N. and Rizzi, M. (2009) The crystal structure of human a-amino-b-carboxymuconate-e-semialdehyde decarboxylase in complex with 1,3-dihydroxyacetonephosphate suggests a regulatory link between NAD synthesis and glycolysis. FEBS J. 276 (22), 6615 6623.

[53] Ganji, S.H., Qin, S., Zhang, L., Kamanna, V.S. and Kashyap, M.L. (2009) Niacin inhibits vascular oxidative stress, redox-sensitive genes, and monocyte adhesion to human aortic endothelial cells. Atherosclerosis 202 (1), 68-75.

[54] Duarte-Pereira, S., Silva, S.S., Azevedo, L., Castro, L., Amorim, A. and Silva, R.M. (2014) NAMPT and NAPRT1: novel polymorphisms and distribution of variants between normal tissues and tumor samples. Sci. Rep. 4, 6311.

[55] Shames, D. S., Elkins, K., Walter, K., Holcomb, T, Du, P., Mohl, D., Xiao, Y., Pham, T., Haverty, P.M., Liederer, B., Liang, X., Yauch, R.L., O’Brien, T., Bourgon, R. Koeppen, H. and Belmont, L.D. (2013) Loss of NAPRT1 expression by tumorspecific promoter methylation provides a novel predictive biomarker for NAMPT inhibitors. Clin. Cancer Res. 9 (24), 6912-6923.

[56] Lasky-Su, J., Anney, R.J., Neale, B.M., Franke, B., Zhou, K., Maller, J.B., Vasquez, A.A., Chen, W., Asherson, P., Buitelaar, J., Banaschewski, T., Ebstein, R., Gill, M., Miranda, A., Mulas, F., Oades, R.D., Roeyers, H., Rothenberger, A., Sergeant, J., Sonuga-Barke, E., Steinhausen, H.C., Taylor, E., Daly, M., Laird, N., Lange, C. and Faraone, S.V. (2008) Genome-wide association scan of the time to onset of attention deficit hyperactivity disorder. Am. J. Med. Genet. B Neuropsychiatr. Genet. 147B (8), 1355-1358.

[57] Cerna, D., Li, H., Flaherty, S., Takebe, N., Coleman, C.N. and Yoo, S.S. (2012) Inhibition of nicotinamide phosphoribosyltransferase (NAMPT) activity by small molecule GMX1778 regulates reactive oxygen species (ROS)-mediated cytotoxicity in a p53- and nicotinic acid phosphoribosyltransferase 1 (NAPRT1)-dependent manner. J. Biol. Chem. 287 (26), 22408-22417. 